DIFFERENTIAL COMPLEXES AND STABILITY OF FINITE ELEMENT METHODS. I. THE DE RHAM COMPLEX

By

Douglas N. Arnold

Richard S. Falk

and

Ragnar Winther

IMA Preprint Series \# 2023

(February 2005)

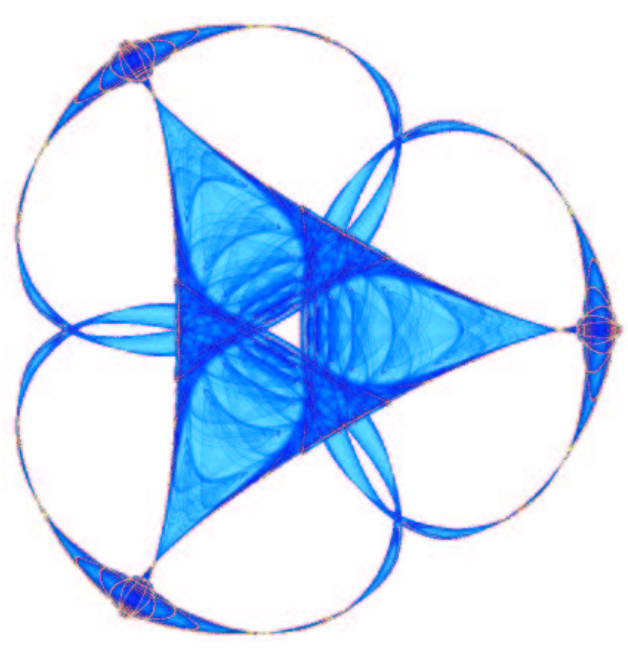

INSTITUTE FOR MATHEMATICS AND ITS APPLICATIONS

UNIVERSITY OF MINNESOTA

514 Vincent Hall

206 Church Street S.E.

Minneapolis, Minnesota 55455-0436

Phone: 612/624-6066 Fax: 612/626-7370

URL: http://www.ima.umn.edu 


\title{
DIFFERENTIAL COMPLEXES AND STABILITY OF FINITE ELEMENT METHODS. I. THE DE RHAM COMPLEX
}

\author{
DOUGLAS N. ARNOLD*, RICHARD S. FALK ${ }^{\dagger}$, AND RAGNAR WINTHER ${ }^{\ddagger}$
}

Abstract. In this paper we explain the relation between certain piecewise polynomial subcomplexes of the de Rham complex and the stability of mixed finite element methods for elliptic problems.

Key words. mixed finite element method, de Rham complex, stability

AMS(MOS) subject classifications. $65 \mathrm{~N} 12,65 \mathrm{~N} 30$

1. Introduction. Many standard finite element methods are based on extremal variational formulations. Typically, the desired solution is characterized as the minimum of some functional over an appropriate trial space of functions, and the discrete solution is then taken to be the minimum of the same functional restricted to a finite dimensional subspace of the trial space consisting of piecewise polynomials with respect to a triangulation of the domain of interest. For such methods, stability is often a simple consideration. For mixed finite element methods, which are based on saddle-point variational principles, the situation is very different: stability is generally a subtle matter and the development of stable mixed finite element methods very challenging. In recent years, a new approach has added greatly to our understanding of stability of mixed methods and enabled the development of stable methods for a number of previously intractable problems. This approach is homological, involving differential complexes related to the problem to be solved, discretizations of these complexes obtained by restricting the differential operators to finite dimensional subspaces, and commutative diagrams relating the two. See, e.g., $[1,2,13,16]$. In this paper we will survey these ideas.

We will concentrate first on the problem of steady state heat conduction. In this problem we seek a scalar temperature field $u$ and a vector flux field $\sigma$ defined on the domain of interest $\Omega \subset \mathbb{R}^{n}$. These satisfy Fourier's law of heat conduction

$$
A \sigma+\operatorname{grad} u=0 \text { on } \Omega
$$

* Institute for Mathematics and its Applications, University of Minnesota, Minneapolis, MN 55442. The work of the first author was supported in part by NSF grant DMS-0411388.

${ }^{\dagger}$ Department of Mathematics, Rutgers University, Hill Center, 110 Frelinghuysen Rd, Rutgers University, Piscataway, NJ 08854-8019. The work of the second author was supported in part by NSF grant DMS03-08347.

${ }^{\ddagger}$ Centre of Mathematics for Applications and Department of Informatics, University of Oslo, P.O. Box 1053, 0316 Oslo, NORWAY. The work of the third author was supported by the Norwegian Research Council. 
and the equation of thermal equilibrium

$$
\operatorname{div} \sigma=f \text { on } \Omega \text {. }
$$

Here $A$ is the thermal resistivity tensor (the inverse of the thermal conductivity tensor), an $n \times n$ symmetric positive-definite matrix field (scalar for an isotropic material), and $f$ the given rate of heat generated per unit volume. To obtain a well-posed problem, these differential equations must be supplemented by suitable boundary conditions, for example, the Dirichlet condition $u=0$ on $\partial \Omega$.

Multiplying the constitutive equation by a test field $\tau$ and integrating by parts over $\Omega$ (taking into account the homogeneous Dirichlet boundary condition), we obtain

$$
\int_{\Omega} A \sigma \cdot \tau d x-\int_{\Omega} u \operatorname{div} \tau d x=0 \quad \forall \tau \in H\left(\operatorname{div}, \Omega ; \mathbb{R}^{n}\right),
$$

while from the equilibrium equation we obtain

$$
\int_{\Omega} \operatorname{div} \sigma v d x=\int_{\Omega} f v d x \quad \forall v \in L^{2}(\Omega) .
$$

The space $H\left(\operatorname{div}, \Omega ; \mathbb{R}^{n}\right)$ consists of all vector fields $\tau: \Omega \rightarrow \mathbb{R}^{n}$ which are square integrable and for which the divergence $\operatorname{div} \tau$ is also square integrable. The pair of spaces $H\left(\operatorname{div}, \Omega ; \mathbb{R}^{n}\right), L^{2}(\Omega)$ are the natural ones for this problem. Indeed, it can be shown that for any $f \in L^{2}(\Omega)$, there is a unique pair $(\sigma, u) \in H\left(\operatorname{div}, \Omega ; \mathbb{R}^{n}\right) \times L^{2}(\Omega)$ satisfying (1.3) and (1.4), and so providing a (weak) solution to (1.1) and (1.2) and the boundary conditions.

Equivalent to the weak formulation is a saddle-point variational formulation, namely

$$
(\sigma, u)=\underset{(\tau, v) \in H(\operatorname{div}) \times L^{2}}{\operatorname{argcrit}}\left[\int\left(\frac{1}{2} A \tau \cdot \tau-v \operatorname{div} \tau\right) d x+\int f v d x\right] .
$$

A more familiar variational characterization of the solution of the heat conduction problem is Dirichlet's principle, which involves the temperature field alone:

$$
u=\underset{v \in \mathscr{\leftrightarrow}^{1}(\Omega)}{\operatorname{argmin}}\left(\frac{1}{2} \int A^{-1} \operatorname{grad} v \cdot \operatorname{grad} v d x-\int f v d x\right) .
$$

This is connected to the second order scalar elliptic equation

$$
-\operatorname{div} A^{-1} \operatorname{grad} u=f \text { on } \Omega
$$

and its natural weak formulation. A standard finite element methods uses a finite element subspace $V_{h}$ of $\stackrel{\circ}{H}^{1}(\Omega)$ and defines the approximate solution

$$
u_{h}=\underset{v \in V_{h}(\Omega)}{\operatorname{argmin}}\left(\frac{1}{2} \int A^{-1} \operatorname{grad} v \cdot \operatorname{grad} v d x-\int f v d x\right) .
$$


Such a method is automatically stable with respect to the $H^{1}$ norm, and consequently the quasioptimal estimate

$$
\left\|u-u_{h}\right\|_{H^{1}} \leq C \inf _{v \in V_{h}}\|u-v\|_{H^{1}}
$$

holds (with $C$ depending only on $A$ and $\Omega$ ).

Returning to the saddle-point formulation, a mixed finite element method defines an approximation solution $\sigma_{h}, u_{h}$ belonging to finite element subspaces $\Sigma_{h} \subset H\left(\operatorname{div}, \Omega ; \mathbb{R}^{n}\right), V_{h} \subset L^{2}(\Omega)$, by

$$
\left(\sigma_{h}, u_{h}\right)=\underset{(\tau, v) \in \Sigma_{h} \times V_{h}}{\operatorname{argcrit}}\left[\int\left(\frac{1}{2} A \tau \cdot \tau-v \operatorname{div} \tau\right) d x+\int f v d x\right] .
$$

The corresponding quasioptimal estimate

$$
\left\|\sigma-\sigma_{h}\right\|_{H(\mathrm{div})}+\left\|u-u_{h}\right\|_{L^{2}} \leq C\left(\inf _{\tau \in \Sigma_{h}}\|\sigma-\tau\|_{H(\mathrm{div})}+\inf _{v \in V_{h}}\|u-v\|_{L^{2}}\right)
$$

will, however, not hold in general. This requires stability, which holds only for very special choices of the finite element spaces. The method (1.6) falls into a well-studied class of saddle-point discretizations for which sufficient (and nearly necessary) conditions for stability can be given $[7,11]$. Namely the discretization will be stable if there exist constants $c_{1}$ and $c_{2}$, independent of the discretization parameter $h$, such that

(A1) $\|\tau\|_{H(\text { div })} \leq c_{1}\|\tau\|_{L^{2}}$ whenever $\tau \in \Sigma_{h}$ satisfies $\int_{\Omega} v \operatorname{div} \tau d x=0$ for all $v \in V_{h}$.

(A2) For all nonzero $v \in V_{h}$, there exists nonzero $\tau \in \Sigma_{h}$ with $\int_{\Omega} v \operatorname{div} \tau d x \geq c_{2}\|\tau\|_{H(\text { div })}\|v\|_{L^{2}}$.

The development of finite element methods satisfying these stability conditions is quite subtle. In the next section, we illustrate this in the simplest case of 1 dimension. In Section 3, we review the two main families of stable finite element spaces for this mixed problem in arbitrary dimensions. Section 4 is a concise review of the main relevant concepts of exterior algebra and exterior calculus, particularly the de Rham complex, the Hodge Laplacian, and the Koszul complex. With these preliminaries, we develop families of finite element discretizations of differential forms of all orders in all dimensions, and show how to combine them into piecewise polynomial subcomplexes of the de Rham complex, obtaining $2^{n-1}$ such subcomplexes in dimension $n$ for each polynomial degree. The finite element spaces involved in these subcomplexes provide most of the stable finite elements that have been derived for mixed problems closely related to a Hodge Laplacian. In the final section, we show how to use these subcomplexes and the commutative diagrams relating them to the de Rham complex to obtain stability of mixed finite element methods. For reasons of space, many results are stated in this paper without proof. Proofs for most of the assertions can be found in the cited references, while for the material new here (the $2^{n-1}$ subcomplexes and the degrees of freedom in (5.1)), a more complete presentation will appear elsewhere. 
2. Some 1-dimensional examples. The subtle nature of stability of finite elements for this problem arises already in the simplest case of one-dimension, with $A \equiv 1$. Thus we are approximating the problem

$$
\sigma+u^{\prime}=0, \quad \sigma^{\prime}=f \text { on }(-1,1), \quad u( \pm 1)=0 .
$$

We shall present some examples to illustrate both stable and unstable choices of elements for this problem. Although in this simple 1-dimensional context, these results can be fully analyzed theoretically, we will limit ourselves to displaying numerical results.

A stable choice of elements in this case consists of continuous piecewise linear functions for the flux and piecewise constant functions for the temperature, which we shall refer to as the $\mathcal{P}_{1}^{\text {cont }}-\mathcal{P}_{0}$ method. The exact and numerical solution are shown in Figure 1 for uniform meshes of 10, 20 , and 40 subintervals, first in the case where $u(x)=1-|x|^{7 / 2}$, and then for the rougher solution $u(x)=1-|x|^{5 / 4}$. In the first case, $u \in H^{3}$ and $\sigma \in H^{2}$, but in the second case, $u \in H^{s}$ and $\sigma \in H^{s-1}$ for $s<7 / 4$ but not larger, which limits the order of convergence of the best approximation by piecewise linears to $\sigma$. In the first part of Table 1 , we see clearly that $\left\|\sigma-\sigma_{h}\right\|_{L^{2}}=O\left(h^{2}\right)$ and $\left\|u-u_{h}\right\|_{L^{2}}=O(h)$, both of which orders are optimal. In the second part of the table, the order of convergence is lowered due to the lowered smoothness of the solution, but the convergence order is as high as that of the best approximation, illustrating the stability of this method.

By contrast, if we use continuous piecewise linear elements for both $\sigma$ and $u$ (e.g., in the hope of improving the order of convergence to $u$ ), the method is not stable. For the smoother problem, $u(x)=1-|x|^{7 / 2}$, we again have second order convergence for $\sigma$ and first order (not second order) convergence for $u$. But for $u(x)=1-|x|^{5 / 4}$, the convergence is clearly wellbelow that of the best approximation, a manifestation of instability, which is plainly visible in Figure 2 and Table 2. This example has been analyzed in detail by Babuška and Narasimhan [5].

In Figure 3, we show the result of using continuous piecewise quadratic elements for $\sigma$ and piecewise constant elements for $u$ (e.g., in hope of improving the order of convergence to $\sigma$ ). The method, which was analyzed in [8], is clearly unstable as well.

Although this $\mathcal{P}_{2}^{\text {cont }}-\mathcal{P}_{0}$ method is not stable, the $\mathcal{P}_{2}^{\text {cont }}-\mathcal{P}_{1}$ method is. In fact, in one dimension the $\mathcal{P}_{r}^{\text {cont }}-\mathcal{P}_{r-1}$ method (continuous piecewise polynomials of degree $r$ for the flux and arbitrary piecewise polynomials of degree $r-1$ for the temperature) is stable for any $r \geq 1$.

3. Basic mixed finite elements in higher dimensions. Consider now the saddle point problem (1.5) in $n$ dimensions and its discretization (1.6) using finite element spaces $\Sigma_{h}$ and $V_{h}$ consisting of piecewise polynomials with respect to a simplicial decomposition $\mathcal{T}_{h}$ of $\Omega$. A simple choice 

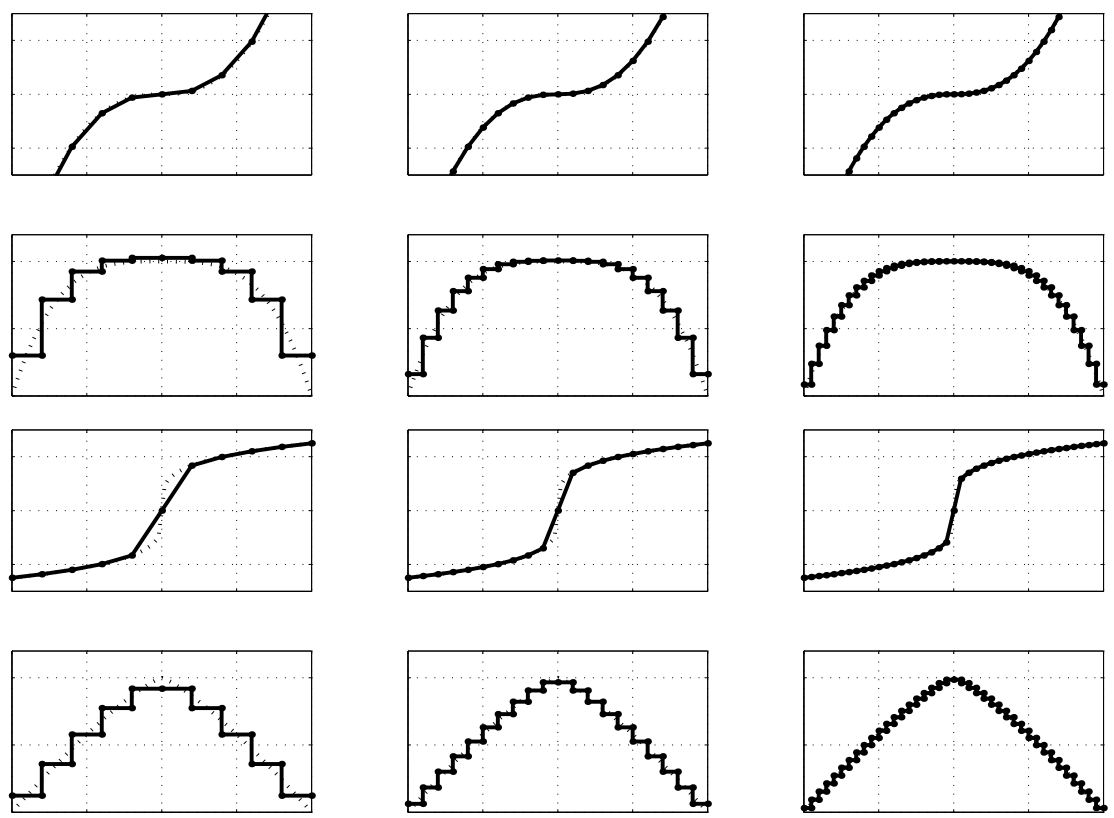

Fig. 1. The $\mathcal{P}_{1}^{\text {cont }}-\mathcal{P}_{0}$ mixed method (which is stable) for meshes of 10, 20, and 40 elements. Lines 1 and 2: $\sigma_{h}$ versus $\sigma$ and $u_{h}$ versus $u$ for the $\mathcal{P}_{1}^{\text {cont }}-\mathcal{P}_{0}$ mixed method when $u(x)=1-|x|^{7 / 2}$. Lines 3 and 4 : same with $u(x)=1-|x|^{5 / 4}$.

of elements, which we saw to be stable in one dimension, is $\mathcal{P}_{1}^{\text {cont }}-\mathcal{P}_{0}$ :

$$
\begin{gathered}
\Sigma_{h}=\left\{\tau \in H^{1}\left(\Omega ; \mathbb{R}^{n}\right)|\tau|_{T} \in \mathcal{P}_{1}(T) \quad \forall T \in \mathcal{T}_{h}\right\}, \\
V_{h}=\left\{v \in L^{2}(\Omega)|v|_{T} \in \mathcal{P}_{0}(T) \quad \forall T \in \mathcal{T}_{h}\right\} .
\end{gathered}
$$

However, for $n>1$, this choice is highly unstable. In fact, on generic triangular meshes the discrete problem is singular and $u_{h}$ is undetermined. And even if $\sigma_{h}$ could be determined, it would belong to the space of divergencefree continuous piecewise linear functions, which reduces to the space of global constants on many triangular meshes, so does not offer any approximation.

However, there are several stable choice of elements in higher dimensions that may be regarded as natural extensions of the simple $\mathcal{P}_{1}^{\text {cont }}-\mathcal{P}_{0}$ element. First, we consider the first order Brezzi-Douglas-Marini elements developed in [10] in two dimensions and [18] and [9] in three dimensions:

$$
\begin{gathered}
\Sigma_{h}=\left\{\tau \in H\left(\operatorname{div}, \Omega ; \mathbb{R}^{n}\right)|\tau|_{T} \in \mathcal{P}_{1}\left(T ; \mathbb{R}^{n}\right) \quad \forall T \in \mathcal{T}_{h}\right\}, \\
V_{h}=\left\{v \in L^{2}(\Omega)|v|_{T} \in \mathcal{P}_{0}(T) \quad \forall T \in \mathcal{T}_{h}\right\} .
\end{gathered}
$$

The difference from the previous choice is that for (3.1), the trial functions for flux are restricted to $H^{1}\left(\Omega ; \mathbb{R}^{n}\right)$, which means that full interelement 
TABLE 1

Errors and orders of convergence for the $\mathcal{P}_{1}^{\text {cont }}-\mathcal{P}_{0}$ mixed method.

\begin{tabular}{|c|c|c|c|c|c|c|}
\hline \multirow[b]{2}{*}{$n$} & \multicolumn{3}{|c|}{$\left\|\sigma-\sigma_{h}\right\|_{L^{2}}$} & \multicolumn{3}{|c|}{$\left\|u-u_{h}\right\|_{L^{2}}$} \\
\hline & err. & $\%$ & order & err. & $\%$ & order \\
\hline 10 & $4.78 \mathrm{e}-02$ & 3.348 & & $1.18 \mathrm{e}-01$ & 10.141 & \\
\hline 20 & $1.20 \mathrm{e}-02$ & 0.838 & 2.00 & $5.87 \mathrm{e}-02$ & 5.027 & 1.01 \\
\hline 40 & $2.99 \mathrm{e}-03$ & 0.210 & 2.00 & $2.93 \mathrm{e}-02$ & 2.508 & 1.00 \\
\hline 80 & $7.49 \mathrm{e}-04$ & 0.052 & 2.00 & $1.46 \mathrm{e}-02$ & 1.253 & 1.00 \\
\hline 160 & $1.87 \mathrm{e}-04$ & 0.013 & 2.00 & $7.31 \mathrm{e}-03$ & 0.627 & 1.00 \\
\hline
\end{tabular}

\begin{tabular}{r|crc|ccc}
\multicolumn{8}{c}{$u=1-|x|^{5 / 4}$} \\
\multicolumn{1}{r}{$n$} & \multicolumn{2}{|c}{$\left\|\sigma-\sigma_{h}\right\|_{L^{2}}$} & \multicolumn{3}{|c}{$\left\|u-u_{h}\right\|_{L^{2}}$} \\
\hline 10 & err. & $\%$ & order & err. & $\%$ & order \\
\hline 20 & $1.75 \mathrm{e}-01$ & 17.102 & & $8.47 \mathrm{e}-02$ & 9.503 & \\
40 & $1.04 \mathrm{e}-01$ & 10.169 & 0.75 & $4.20 \mathrm{e}-02$ & 4.712 & 1.01 \\
80 & $3.17 \mathrm{e}-02$ & 6.047 & 0.75 & $2.09 \mathrm{e}-02$ & 2.349 & 1.00 \\
160 & $2.18 \mathrm{e}-02$ & 3.595 & 0.75 & $1.04 \mathrm{e}-02$ & 1.173 & 1.00 \\
\hline
\end{tabular}

continuity must be imposed (a piecewise polynomial belongs to $H^{1}$ if and only if it is continuous). But for the stable choice (3.2), the flux functions need only belong to $H($ div), which requires only interelement continuity of the normal component (a piecewise polynomial vector field belongs to $H$ (div) if and only if its normal component is continuous across each $(n-1)$ dimensional face shared by two elements).

In order that the spaces given in (3.2) are implementable via the standard finite element assembly procedure - in fact, in order that they that are finite element spaces in the sense of [12]—we must be able to specify degrees of freedom for the local shape function spaces $\mathcal{P}_{1}\left(T ; \mathbb{R}^{n}\right)$ and $\mathcal{P}_{0}(T)$ which enforce exactly the required interelement continuity. For the former, we choose the moments of degree at most 1 of the normal component of the field on each face of the element:

$$
\tau \mapsto \int_{f}(\tau \cdot n) p, \quad p \in \mathcal{P}_{1}(f), \quad f \in \Delta_{n-1}(T) .
$$

(We use the notation $\Delta_{k}(T)$ to denote the set of subsimplices of dimension $k$ of the simplex $T$, i.e., the set of vertices for $k=0$, edges for $k=1$, etc.) Since the normal component of the field is itself linear, these functionals exactly impose the desired continuity of the normal component. Choosing 

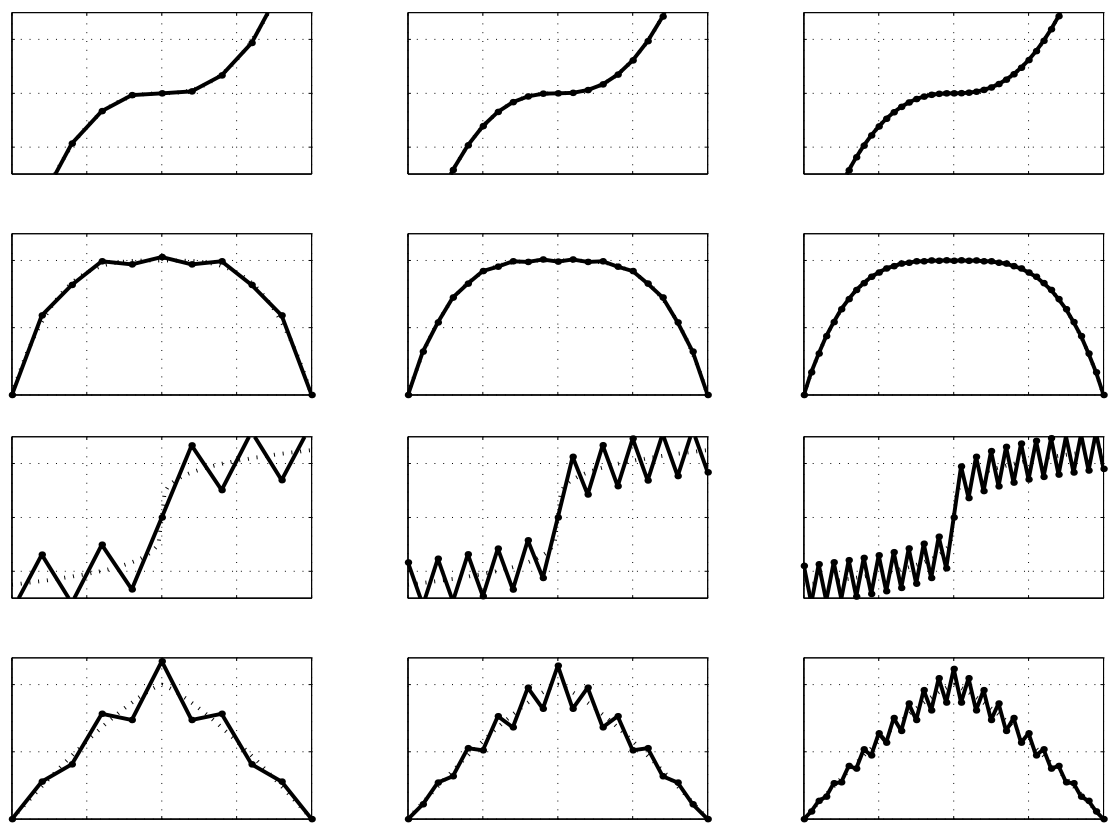

FIG. 2. $\mathcal{P}_{1}^{\text {cont }} \mathcal{P}_{1}^{\text {cont }}$ mixed method (unstable) for meshes of 10, 20, and 40 elements.

a basis for each of the $n$-dimensional spaces $\mathcal{P}_{1}(f)$ for each of $n+1$ faces $f \in \Delta_{n-1}(T)$, we obtain $n(n+1)=\operatorname{dim} P_{1}\left(T ; \mathbb{R}^{n}\right)$ degrees of freedom. These degrees of freedom are clearly unisolvent, since if they all vanish for some $\tau \in \mathcal{P}_{1}(T)$, then at each vertex $\tau \cdot n$ vanishes for the vector $n$ normal to each face meeting at the vertex. These normal vectors span $\mathbb{R}^{n}$, so $\tau$ itself vanishes at each vertex, and therefore vanishes on all of $T$. Since the space $V_{h}$ involves no interelement continuity, we make the obvious choice of degrees of freedom for $\mathcal{P}_{0}(T)$ :

$$
v \mapsto \int_{T} v w, \quad w \in \mathcal{P}_{0}(T)
$$

The degrees of freedom (3.3) determine a projection operator $\Pi_{\Sigma_{h}}$ : $H^{1}\left(\Omega ; \mathbb{R}^{n}\right) \rightarrow \Sigma_{h}$ onto the first-order Brezzi-Douglas-Marini space, given by

$$
\int_{f} \Pi_{\Sigma_{h}}(\tau \cdot n) p=\int_{f}(\tau \cdot n) p, \quad p \in \mathcal{P}_{1}(f), \quad f \in \Delta_{n-1}(T),
$$

while the projection operator $\Pi_{V_{h}}: L^{2}(\Omega) \rightarrow V_{h}$ determined by the degrees of freedom (3.4) is simply the usual $L^{2}$-projection. An important relation between these operators is expressed by the commutativity of the following 
TABLE 2

Errors and orders of convergence for the $\mathcal{P}_{1}^{\text {cont }}-\mathcal{P}_{1}^{\text {cont }}$ mixed method.

\begin{tabular}{r|ccc|crc}
\multicolumn{8}{c}{$u=1-|x|^{7 / 2}$} \\
\hline & \multicolumn{3}{|c|}{$\left\|\sigma-\sigma_{h}\right\|_{L^{2}}$} & \multicolumn{3}{|c}{$\left\|u-u_{h}\right\|_{L^{2}}$} \\
\cline { 2 - 6 } & err. & $\%$ & order & \multicolumn{1}{|c}{ err. } & \multicolumn{1}{c}{$\%$} & order \\
\hline 10 & $2.09 \mathrm{e}-02$ & 1.464 & & $2.38 \mathrm{e}-01$ & 20.429 & \\
20 & $5.07 \mathrm{e}-03$ & 0.355 & 2.04 & $1.17 \mathrm{e}-01$ & 10.066 & 1.02 \\
40 & $1.25 \mathrm{e}-03$ & 0.088 & 2.02 & $5.85 \mathrm{e}-02$ & 5.011 & 1.01 \\
80 & $3.11 \mathrm{e}-04$ & 0.022 & 2.01 & $2.92 \mathrm{e}-02$ & 2.502 & 1.00 \\
160 & $7.76 \mathrm{e}-05$ & 0.005 & 2.00 & $1.46 \mathrm{e}-02$ & 1.251 & 1.00 \\
\hline
\end{tabular}

\begin{tabular}{r|ccc|ccc}
\multicolumn{8}{c}{$u=1-|x|^{5 / 4}$} \\
\hline & \multicolumn{3}{|c|}{$\left\|\sigma-\sigma_{h}\right\|_{L^{2}}$} & \multicolumn{3}{|c}{$\left\|u-u_{h}\right\|_{L^{2}}$} \\
$n$ & err. & $\%$ & order & err. & $\%$ & order \\
\hline 10 & $3.96 \mathrm{e}-01$ & 38.769 & & $2.24 \mathrm{e}-01$ & 25.182 & \\
20 & $3.36 \mathrm{e}-01$ & 32.875 & 0.24 & $1.42 \mathrm{e}-01$ & 15.974 & 0.66 \\
40 & $2.83 \mathrm{e}-01$ & 27.759 & 0.24 & $1.04 \mathrm{e}-01$ & 11.663 & 0.45 \\
80 & $2.39 \mathrm{e}-01$ & 23.391 & 0.25 & $8.23 \mathrm{e}-02$ & 9.243 & 0.34 \\
160 & $2.01 \mathrm{e}-01$ & 19.689 & 0.25 & $6.77 \mathrm{e}-02$ & 7.601 & 0.28 \\
\hline
\end{tabular}

diagram:

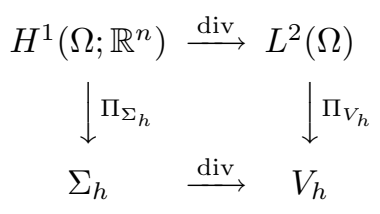

(This can be verified via integration by parts.) Note that we have taken $H^{1}\left(\Omega ; \mathbb{R}^{n}\right)$, rather than $H\left(\operatorname{div}, \Omega ; \mathbb{R}^{n}\right)$, as the domain of $\Pi_{\Sigma_{h}}$. This is because $\Pi_{\Sigma_{h}}$ defines a bounded operator $H^{1}\left(\Omega ; \mathbb{R}^{n}\right) \rightarrow L^{2}\left(\Omega ; \mathbb{R}^{n}\right)$. In fact, it is bounded uniformly in the mesh size $h$ if we restrict to a shape-regular family of triangulations. However, $\Pi_{\Sigma_{h}}$ does not extend to a bounded operator on all of $H\left(\operatorname{div}, \Omega ; \mathbb{R}^{n}\right)$, because of lack of sufficiently regular traces.

The commutative diagram (3.5) encapsulates the properties of of the spaces $\Sigma_{h}$ and $V_{h}$ needed to verify the stability conditions (A1) and (A2). First, since $\operatorname{div} \Sigma_{h} \subset V_{h}$, any $\tau \in \Sigma_{h}$ satisfying $\int_{\Omega} v \operatorname{div} \tau d x=0$ for all $v \in V_{h}$ is in fact divergence-free, and so (A1) holds. In order to verify (A2), let $v \in V_{h}$ be given. Since div maps $H^{1}\left(\Omega ; \mathbb{R}^{n}\right)$ onto $L^{2}(\Omega)$ and admits a bounded right inverse, c.f. [14], we can find $\tilde{\tau} \in H^{1}\left(\Omega ; \mathbb{R}^{n}\right)$ with $\operatorname{div} \tilde{\tau}=v$ and $\|\tilde{\tau}\|_{H^{1}} \leq c\|v\|_{L^{2}}$. Now let $\tau=\Pi_{\Sigma_{h}} \tilde{\tau}$. From the commutative 

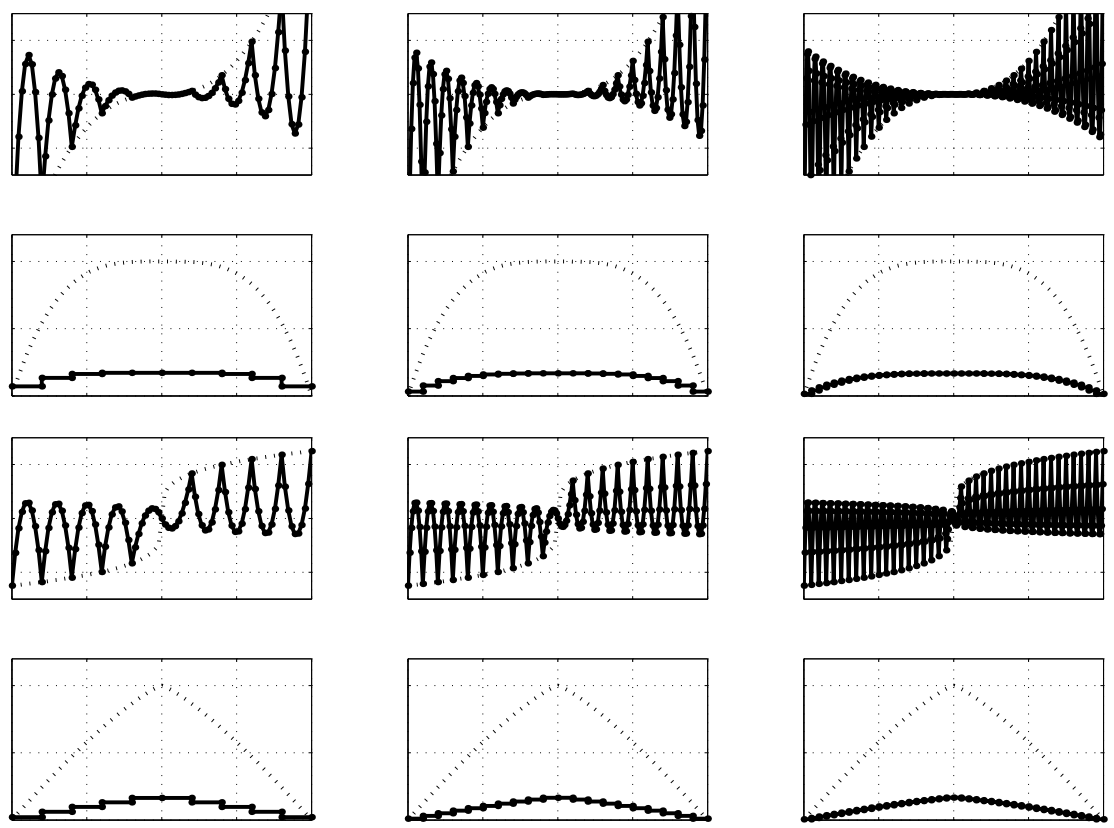

FIG. 3. $\mathcal{P}_{2}^{\text {cont }}-\mathcal{P}_{0}$ mixed method (unstable).

diagram (3.5) we see that

$$
\operatorname{div} \tau=\operatorname{div} \Pi_{\Sigma_{h}} \tilde{\tau}=\Pi_{V_{h}} \operatorname{div} \tilde{\tau}=\Pi_{V_{h}} v=v .
$$

Invoking also the $H^{1}\left(\Omega ; \mathbb{R}^{n}\right) \rightarrow L^{2}(\Omega)$ boundedness of $\Pi_{\Sigma_{h}}$, we obtain

$$
\int_{\Omega} v \operatorname{div} \tau d x=\|v\|_{L^{2}}^{2}, \quad\|\tau\|_{H(\operatorname{div})} \leq c^{\prime}\|v\|_{L^{2}},
$$

from which (A2) follows. Thus the first-order Brezzi-Douglas-Marini elements (3.2) are stable in $n$ dimensions.

Note that (3.2) coincides with (3.1) in the case $n=1$, so these elements are indeed a generalization to higher dimensions of the simple $\mathcal{P}_{1}^{\text {cont }}-\mathcal{P}_{0}$ elements. Moreover, they can be viewed as the lowest order case of a family of stable elements of arbitrary order:

$$
\begin{gathered}
\Sigma_{h}=\left\{\tau \in H\left(\operatorname{div}, \Omega ; \mathbb{R}^{n}\right)|\tau|_{T} \in \mathcal{P}_{r}\left(T ; \mathbb{R}^{n}\right) \quad \forall T \in \mathcal{T}_{h}\right\}, \\
V_{h}=\left\{v \in L^{2}(\Omega)|v|_{T} \in \mathcal{P}_{r-1}(T) \quad \forall T \in \mathcal{T}_{h}\right\} .
\end{gathered}
$$

The degrees of freedom are

$$
\tau \mapsto \int_{f}(\tau \cdot n) p, \quad p \in \mathcal{P}_{r}(f), \quad f \in \Delta_{n-1}(T),
$$


and an additional $(r-1)\left(\begin{array}{c}n+r-1 \\ r\end{array}\right)$ moments over $T$, about which more will be said below. In one dimension, this is just the $\mathcal{P}_{r}^{\text {cont }}-\mathcal{P}_{r-1}$ element discussed at the end of the last section. The first two elements in the Brezzi-DouglasMarini family in two dimensions are pictured in Figure 4.
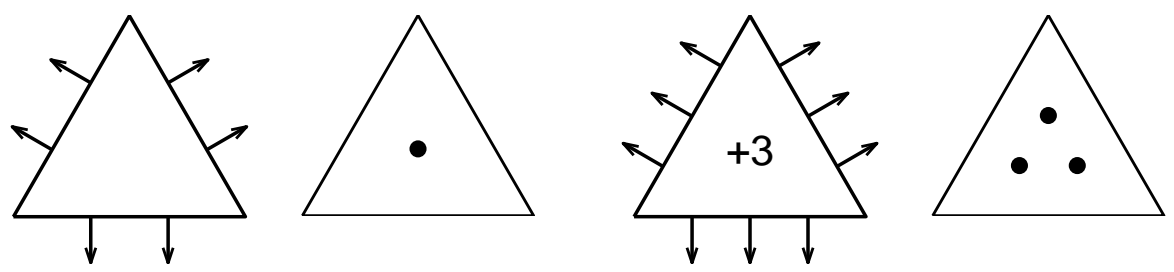

FIG. 4. Brezzi-Douglas-Marini element pairs for $r=1$ and 2 in two dimensions.

Although the Brezzi-Douglas-Marini family of elements provide a natural analogue of the $\mathcal{P}_{r}^{\text {cont }}-\mathcal{P}_{r-1}$ family of elements to higher dimensions, it is not the only such analogue. Another is the Raviart-Thomas family introduced in [19] and improved and extended from two to three dimensions in [17]. To describe it, we define for $T \subset \mathbb{R}^{n}$ and integer $r \geq 0$,

$\mathcal{R} \mathcal{T}_{r}(T)=\left\{\tau: T \rightarrow \mathbb{R}^{n} \mid \tau(x)=\alpha(x)+x \beta(x), \alpha \in \mathcal{P}_{r}\left(T ; \mathbb{R}^{n}\right), \beta \in \mathcal{P}_{r}(T)\right\}$.

Then the Raviart-Thomas elements of index $r \geq 0$ are

$$
\begin{gathered}
\Sigma_{h}=\left\{\tau \in H\left(\operatorname{div}, \Omega ; \mathbb{R}^{n}\right)|\tau|_{T} \in \mathcal{R} \mathcal{T}_{r}(T) \quad \forall T \in \mathcal{T}_{h}\right\}, \\
V_{h}=\left\{v \in L^{2}(\Omega)|v|_{T} \in \mathcal{P}_{r}(T) \quad \forall T \in \mathcal{T}_{h}\right\},
\end{gathered}
$$

with some element diagrams show in Figure 5. These elements are defined in all dimensions. In dimension one, the Raviart-Thomas elements (3.8) coincide with the Brezzi-Douglas-Marini elements (3.6) with $r$ replaced by $r+1$. But for $n \geq 2$, these families are distinct.
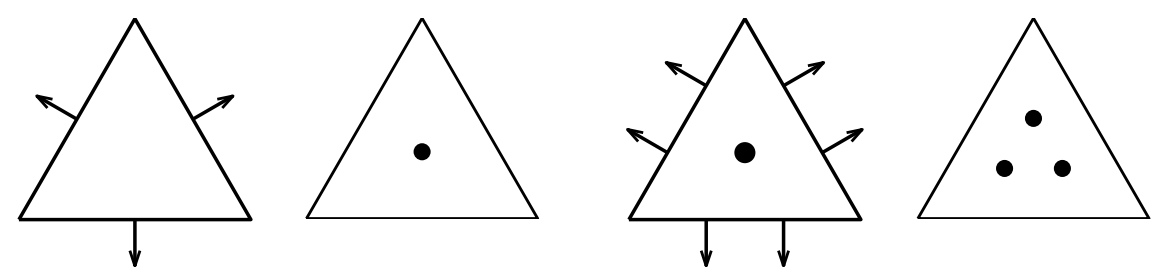

FIG. 5. Raviart-Thomas element pairs for $r=0$ and 1 in two dimensions.

4. Exterior calculus. The finite elements described above, and others, can better be understood with the help of differential forms and exterior calculus. We begin by recalling the basic notions of exterior algebra. (For details see, e.g., [3], Ch. 7. Let $V$ be a vector space of dimension $n$. We denote by $\operatorname{Alt}^{k} V$ the space of exterior $k$-forms on $V$, i.e., of alternating 
$k$-linear maps $V \times \cdots \times V \rightarrow \mathbb{R}$. That is, an element of $\mathrm{Alt}^{k} V$ assigns a real number to $k$ elements of $V$, is linear in each argument, and reverses sign when two arguments are interchanged. In particular, $\operatorname{Alt}^{1} V$ is simply the dual space $V^{*}$ and $\operatorname{Alt}^{0} V$ may be identified with $\mathbb{R}$. For $k>n$, $\operatorname{Alt}^{k} V=0$, while for all $k$ we have

$$
\operatorname{dim} \mathrm{Alt}^{k} V=\left(\begin{array}{l}
n \\
k
\end{array}\right)
$$

A form in the one-dimensional space $\mathrm{Alt}^{n} V$ is uniquely determined by its value on any one coordinate frame (i.e., ordered basis). The value of the form on any other ordered $n$-tuple of vectors can be obtained by expanding the vectors in the coordinate frame to obtain a matrix, and multiplying the value of the form on the coordinate frame by the determinant of the matrix.

An inner product on $V$ determines an inner product on each $\mathrm{Alt}^{k} V$ by the formula

$$
\langle\omega, \eta\rangle=\sum_{1 \leq \sigma_{1}<\cdots<\sigma_{k} \leq n} \omega\left(v_{\sigma_{1}}, \ldots, v_{\sigma_{k}}\right) \eta\left(v_{\sigma_{1}}, \ldots, v_{\sigma_{k}}\right), \quad \omega, \eta \in \mathrm{Alt}^{k} V .
$$

We recall also the exterior product $\wedge: \mathrm{Alt}^{j} V \times \mathrm{Alt}^{k} V \rightarrow \mathrm{Alt}^{j+k} V$ defined by

$$
\begin{array}{r}
(\omega \wedge \eta)\left(v_{1}, \ldots, v_{j+k}\right)=\sum_{\sigma \in \Sigma(j, j+k)}(\operatorname{sign} \sigma) \omega\left(v_{\sigma_{1}}, \ldots, v_{\sigma_{j}}\right) \eta\left(v_{\sigma_{j+1}}, \ldots, v_{\sigma_{j+k}}\right), \\
\omega \in \operatorname{Alt}^{j} V, v_{i} \in V,
\end{array}
$$

where $\Sigma(j, j+k)$ is the set of all permutations of $\{1, \ldots, j+k\}$, for which $\sigma_{1}<\sigma_{2}<\cdots \sigma_{j}$ and $\sigma_{j+1}<\sigma_{j+2}<\cdots \sigma_{j+k}$.

In the case $V=\mathbb{R}^{n}$, there is a canonical basis, and we denote by $d x^{1}, \ldots, d x^{n}$ the elements of the dual basis, which form a canonical basis for $\mathrm{Alt}^{1} \mathbb{R}^{n}$. Then a canonical basis for $\mathrm{Alt}^{k} \mathbb{R}^{n}$ consists of the forms $d x^{\sigma_{1}} \wedge$ $\cdots \wedge d x^{\sigma_{k}}$, where $1 \leq \sigma_{1}<\cdots<\sigma_{k} \leq n$.

For readers less familiar with exterior algebra, it is worthwhile to examine in detail the example $V=\mathbb{R}^{3}$, endowed with the usual inner product and orientation. In this case

- The general element of $\mathrm{Alt}^{0} \mathbb{R}^{3}$ is $c, c \in \mathbb{R}$.

- The general element of $\operatorname{Alt}^{1} \mathbb{R}^{3}$ is $\langle u, \cdot\rangle$, or, equivalently, $u_{1} d x^{1}+u_{2} d x^{2}+u_{3} d x^{3}, u \in \mathbb{R}^{3}$.

- The general element of $\mathrm{Alt}^{2} \mathbb{R}^{3}$ is $\langle w, \cdot \times \cdot\rangle$, or, equivalently, $w_{1} d x^{2} \wedge d x^{3}-w_{2} d x^{1} \wedge d x^{3}+w_{3} d x^{1} \wedge d x^{2}, w \in \mathbb{R}^{3}$.

- The general element of $\mathrm{Alt}^{3} \mathbb{R}^{3}$ is $g\langle\cdot, \cdot \times \cdot\rangle$, or, equivalently, $g d x^{1} \wedge d x^{2} \wedge d x^{3}, g \in \mathbb{R}$.

Thus we may identify Alt ${ }^{0} \mathbb{R}^{3}$ and $\mathrm{Alt}^{3} \mathbb{R}^{3}$ with $\mathbb{R}$ and $\mathrm{Alt}^{1} \mathbb{R}^{3}$ and $\mathrm{Alt}^{2} \mathbb{R}^{3}$ with $\mathbb{R}^{3}$. 
Next we identify the exterior product $\mathrm{Alt}^{j} \mathbb{R}^{3} \times \mathrm{Alt}^{k} \mathbb{R}^{3} \rightarrow \mathrm{Alt}^{j+k} \mathbb{R}^{3}$ for $0 \leq j \leq k, j+k \leq 3$. (The exterior product for other values of $j, k$ either follows from these or is identically zero.) If $j=0$, the exterior product is the ordinary scalar multiplication. The exterior product $\mathrm{Alt}^{1} \mathbb{R}^{3} \times \mathrm{Alt}^{1} \mathbb{R}^{3} \rightarrow$ $\mathrm{Alt}^{2} \mathbb{R}^{3}$ corresponds under our identifications to the usual cross product $\mathbb{R}^{3} \times \mathbb{R}^{3} \rightarrow \mathbb{R}^{3}$. Finally, the exterior product $\mathrm{Alt}^{1} \mathbb{R}^{3} \times \mathrm{Alt}^{2} \mathbb{R}^{3} \rightarrow \mathrm{Alt}^{3} \mathbb{R}^{3}$ corresponds to the usual inner product $\mathbb{R}^{3} \times \mathbb{R}^{3} \rightarrow \mathbb{R}$. It is straightforward to check that given the identifications mentioned, the inner product defined above on $\mathrm{Alt}^{k} \mathbb{R}^{3}$ is the usual product in $\mathbb{R}$ for $k=0$ or 3 , and the Euclidean product in $\mathbb{R}^{3}$ for $k=1$ or 2 .

Having reviewed the basic definitions of exterior algebra, we now turn to exterior calculus. If $\Omega$ is any smooth manifold, we define a smooth differential $k$-form on $\Omega$ as a mapping $\omega$ which assigns to each $x \in \Omega$ an alternating linear form $\omega_{x} \in \operatorname{Alt}^{k}\left(T_{x} \Omega\right)$ on the tangent space $T_{x} \Omega$ to $\Omega$ at $x$. We denote the space of all smooth differential $k$-forms on $\Omega$ by $\Lambda^{k}(\Omega)$. We write $C^{0} \Lambda^{k}(\Omega)$ to denote the larger space of all continuous differential forms, and use a similar notation for other functional spaces. For example, if $\Omega$ is a Riemannian manifold, we can talk about $L^{2} \Lambda^{k}(\Omega)$, etc.

Differential forms can be integrated and differentiated without the need for any additional structure, such as a measure or metric, on the manifold $\Omega$. If $0 \leq k \leq n$ is an integer, $f$ is an oriented piecewise smooth $k$ dimensional submanifold of $\Omega$, and $\omega$ is a $k$-form, then the integral $\int_{f} \omega \in \mathbb{R}$ is well-defined. Thus, for example, 0 -forms can be evaluated at points, 1 -forms can be integrated on curves, and 2-forms can be integrated over surfaces. Also, for each such $k$, the exterior derivative $d_{k}$ is a linear operator mapping $\Lambda^{k}(\Omega)$ into $\Lambda^{k+1}(\Omega)$. The de Rham complex of $\Omega$ is the sequence of maps

$$
\mathbb{R} \hookrightarrow \Lambda^{0}(\Omega) \stackrel{d}{\longrightarrow} \Lambda^{1}(\Omega) \stackrel{d}{\longrightarrow} \cdots \stackrel{d}{\longrightarrow} \Lambda^{n}(\Omega) \rightarrow 0
$$

where we have followed the usual convention of suppressing the subscript on the $d_{k}$. This is a complex in the sense that the composition of two consecutive maps is zero $\left(d_{k} d_{k-1}=0\right)$, and we can consider the $k$ th de Rham cohomology space, defined to be the quotient of the null space of $d_{k}$ modulo the range of $d_{k-1}$. If the manifold is contractible, this complex is exact in the sense that the cohomology spaces all vanish, or, equivalently, the range of each map is precisely equal to (and not just contained in) the null space of the succeeding map.

Assuming that $\Omega$ is a Riemannian manifold, so each tangent space $T_{x} \Omega$ is endowed with an inner product, we have an inner product on each $\Lambda^{k}(\Omega)$ which can be completed to a Hilbert space $L^{2} \Lambda^{k}(\Omega)$. We can define the Sobolev space of differential $k$-forms:

$$
H \Lambda^{k}(\Omega)=\left\{\omega \in L^{2} \Lambda^{k}(\Omega) \mid d \omega \in L^{2} \Lambda^{k+1}(\Omega)\right\} .
$$

The $L^{2}$ de Rham complex

$$
\mathbb{R} \hookrightarrow H \Lambda^{0}(\Omega) \stackrel{d}{\longrightarrow} H \Lambda^{1}(\Omega) \stackrel{d}{\longrightarrow} \cdots \stackrel{d}{\longrightarrow} H \Lambda^{n}(\Omega) \rightarrow 0
$$


has the same cohomology as the smooth de Rham complex.

Viewing $d_{k}$ as a (closed, densely-defined) unbounded linear operator mapping $L^{2} \Lambda^{k}(\Omega)$ to $L^{2} \Lambda^{k+1}(\Omega)$ with domain $H \Lambda^{k}(\Omega)$, we may use the inner product of differential forms to define the adjoint $d_{k}^{*}$ which maps a dense subspace of $L^{2} \Lambda^{k+1}(\Omega)$ to $L^{2} \Lambda^{k}(\Omega)$. Namely, $\omega \in L^{2} \Lambda^{k+1}(\Omega)$ belongs to the domain of $d_{k}^{*}$ if there exists $d_{k}^{*} \omega \in L^{2} \Lambda^{k}(\Omega)$ such that

$$
\left\langle d^{*} \omega, \eta\right\rangle_{L^{2} \Lambda^{k}}=\langle\omega, d \eta\rangle_{L^{2} \Lambda^{k+1}}, \quad \eta \in H \Lambda^{k}(\Omega) .
$$

The Hodge Laplacian is then the map $d^{*} d+d d^{*}$ (or, more precisely, $d_{k}^{*} d_{k}+$ $\left.d_{k-1} d_{k-1}^{*}\right)$ which maps a part of $L^{2} \Lambda^{k}(\Omega)$ into $L^{2} \Lambda^{k}(\Omega)$.

In case $\Omega$ is an open subset of $\mathbb{R}^{n}$, every differential $k$-form may be written uniquely in the form

$$
\omega_{x}=\sum_{i_{1}<\cdots<i_{k}} a_{i_{1} \ldots i_{k}}(x) d x^{i_{1}} \wedge \cdots \wedge d x^{i_{k}},
$$

for some smooth functions $a_{i_{1} \ldots i_{k}}: \Omega \rightarrow \mathbb{R}$. This is useful for computing the exterior derivative since:

$$
d\left(a d x^{i_{1}} \wedge \cdots \wedge d x^{i_{k}}\right)=\sum_{j=1}^{n} \frac{\partial a}{\partial x^{j}} d x^{j} \wedge d x^{i_{1}} \wedge \cdots \wedge d x^{i_{k}} .
$$

For use later in the paper, we introduce the Sobolev space $H^{s} \Lambda^{k}(\Omega)$ consisting of differential forms of the form (4.3) for which the coefficients $a_{i_{1} \ldots i_{k}} \in$ $H^{s}(\Omega)$. The corresponding norm is given by $\|\omega\|_{s}=\left(\sum\left\|a_{i_{1} \ldots i_{k}}\right\|_{s}^{2}\right)^{1 / 2}$, which we write simply as $\|\omega\|$ if $s=0$.

For $\Omega \subset \mathbb{R}^{n}$, we can also define the notion of polynomial differential forms. Namely, we say that $\omega \in \Lambda^{k}(\Omega)$ is a (homogeneous) polynomial $k$-form of degree $r$ if for any choice $v^{1}, \ldots, v^{k} \in \mathbb{R}^{n}$, the map

$$
x \mapsto \omega_{x}\left(v^{1}, \ldots, v^{k}\right)
$$

is the restriction to $\Omega$ of a (homogeneous) polynomial of degree $r$. For $\omega$ given by (4.3), this is equivalent to saying that each of the coefficients $a_{i_{1} \ldots i_{k}}$ is a (homogeneous) polynomial of degree $r$. We denote the spaces of polynomial $k$-forms of degree $r$ and homogeneous $k$-forms of degree $r$ by $\mathcal{P}_{r} \Lambda^{k}(\Omega)$ and $\mathcal{H}_{r} \Lambda^{k}(\Omega)$, respectively. We shall verify below that the polynomial de Rham complex

$$
\mathbb{R} \hookrightarrow \mathcal{P}_{r} \Lambda^{0}(\Omega) \stackrel{d}{\longrightarrow} \mathcal{P}_{r-1} \Lambda^{1}(\Omega) \stackrel{d}{\longrightarrow} \cdots \stackrel{d}{\longrightarrow} \mathcal{P}_{r-n} \Lambda^{n}(\Omega) \rightarrow 0
$$

is exact for every $r \geq 0$ (with the understanding that $\mathcal{H}_{m}=\mathcal{P}_{m}=0$ for $m<0$ ). The same is true for the homogeneous polynomial de Rham sequence

$$
R \hookrightarrow \mathcal{H}_{r} \Lambda^{0}(\Omega) \stackrel{d}{\longrightarrow} \mathcal{H}_{r-1} \Lambda^{1}(\Omega) \stackrel{d}{\longrightarrow} \cdots \stackrel{d}{\longrightarrow} \mathcal{H}_{r-n} \Lambda^{n}(\Omega) \rightarrow 0
$$


where $R=\mathbb{R}$ if $r=0$ and $R=0$ otherwise.

Finally, still in the case $\Omega \subset \mathbb{R}^{n}$, we introduce the Koszul differential $\kappa=\kappa_{k}: \Lambda^{k} \rightarrow \Lambda^{k-1}$, defined by

$$
(\kappa \omega)_{x}\left(v^{1}, \ldots, v^{k-1}\right)=\omega_{x}\left(x, v^{1}, \ldots, v^{k-1}\right) .
$$

Note that $\kappa_{k-1} \kappa_{k}=0$. Also, $\kappa$ maps $\mathcal{H}_{r} \Lambda^{k}(\Omega)$ into $\mathcal{H}_{r+1} \Lambda^{k-1}(\Omega)$, i.e., the Koszul differential increases polynomial degree and decreases the order of the differential form, exactly the opposite of exterior differentiation, which maps $\mathcal{H}_{r+1} \Lambda^{k-1}(\Omega)$ into $\mathcal{H}_{r} \Lambda^{k}(\Omega)$. The two operations are connected by the formula

$$
(d \kappa+\kappa d) \omega=(r+k) \omega, \quad \omega \in \mathcal{H}_{r} \Lambda^{k}(\Omega) .
$$

This can be used to establish exactness of the homogeneous polynomial de Rham sequence (4.5), and also of the homogeneous Koszul complex

$0 \rightarrow \mathcal{H}_{r-n} \Lambda^{n}(\Omega) \stackrel{\kappa}{\longrightarrow} \mathcal{H}_{r-n+1} \Lambda^{n-1}(\Omega) \stackrel{\kappa}{\longrightarrow} \cdots \stackrel{\kappa}{\longrightarrow} \mathcal{H}_{r} \Lambda^{0}(\Omega) \rightarrow R \rightarrow 0$

where again $R=\mathbb{R}$ if $r=0$ and $R=0$ otherwise. Adding over polynomial degrees we get the exactness of (4.4) and of the Koszul complex

$$
0 \rightarrow \mathcal{P}_{r-n} \Lambda^{n}(\Omega) \stackrel{\kappa}{\longrightarrow} \mathcal{P}_{r-n+1} \Lambda^{n-1}(\Omega) \stackrel{\kappa}{\longrightarrow} \cdots \stackrel{\kappa}{\longrightarrow} \mathcal{P}_{r} \Lambda^{0}(\Omega) \rightarrow \mathbb{R} \rightarrow 0
$$

We use the Koszul differential to define an important space of polynomial forms on a domain $T \subset \mathbb{R}^{n}$ :

$$
\mathcal{P}_{r}^{+} \Lambda^{k}(T)=\mathcal{P}_{r} \Lambda^{k}(T)+\kappa \mathcal{P}_{r} \Lambda^{k+1}(T),
$$

where $\kappa$ is the Koszul differential defined in (4.6). Clearly

$$
\mathcal{P}_{r}^{+} \Lambda^{k}(T)=\mathcal{P}_{r} \Lambda^{k}(T)+\kappa \mathcal{H}_{r} \Lambda^{k+1}(T)
$$

and, in view of (4.7),

$$
\mathcal{P}_{r}^{+} \Lambda^{k}(T)+d \mathcal{H}_{r+2} \Lambda^{k-1}=\mathcal{P}_{r+1} \Lambda^{k}(T) .
$$

For 0 -forms and $n$-forms, the $\mathcal{P}^{+}$spaces are nothing new:

$$
\mathcal{P}_{r}^{+} \Lambda^{0}(T)=\mathcal{P}_{r+1} \Lambda^{0}(T), \quad \mathcal{P}_{r}^{+} \Lambda^{n}(T)=\mathcal{P}_{r} \Lambda^{n}(T) .
$$

However, for $0<k<n$

$$
\mathcal{P}_{r} \Lambda^{k}(T) \subsetneq \mathcal{P}_{r}^{+} \Lambda^{k}(T) \subsetneq \mathcal{P}_{r+1} \Lambda^{k}(T) .
$$

If we identify $\Lambda^{n-1}(T)$ with $C^{\infty}\left(T ; \mathbb{R}^{n}\right)$, then $\mathcal{P}_{r}^{+} \Lambda^{n-1}(T)$ corresponds exactly to the space of Raviart-Thomas polynomial fields defined in (3.7). In the general case, we may compute their dimensions:

$$
\operatorname{dim} \mathcal{P}_{r}^{+} \Lambda^{k}(T)=\left(\begin{array}{c}
n+r \\
n
\end{array}\right)\left(\begin{array}{l}
n \\
k
\end{array}\right)+\left(\begin{array}{c}
n+r \\
n-k-1
\end{array}\right)\left(\begin{array}{c}
r+k \\
k
\end{array}\right)
$$


while

$$
\operatorname{dim} \mathcal{P}_{r} \Lambda^{k}(T)=\left(\begin{array}{c}
n+r \\
n
\end{array}\right)\left(\begin{array}{l}
n \\
k
\end{array}\right)
$$

Finally, we specialize to the case $\Omega \subset \mathbb{R}^{3}$. Then $T_{x} \mathbb{R}^{3} \cong \mathbb{R}^{3}$ and Alt ${ }^{k} T_{x} \mathbb{R}^{3}$ may be identified with $\mathbb{R}$ for $k=0,3$ and with $\mathbb{R}^{3}$ for $k=1,2$. We can then interpret the integral in the sense of differential forms as follows. If $\omega$ is a 0 -form, and $v$ a point in $\Omega$, then $\int_{v} \omega=\omega(v)$. If $\omega$ is a function on $\Omega$ which we identify with a 1 -form and $e$ is an oriented curve in $\Omega$, then the differential form integral $\int_{e} \omega=\int_{e} \omega \cdot t d \mathfrak{H}_{1}$ where $t$ is the unit tangent to $e$ (determined uniquely by the orientation) and $\mathfrak{H}_{1}$ is 1 dimensional Hausdorff measure. If $\omega$ is a function on $\Omega$ which we identify with a 2 -form and $f$ is an oriented surface in $\Omega$, then the differential form integral $\int_{f} \omega=\int_{f} \omega \cdot n d \mathfrak{H}_{2}$ where $n$ is the unit normal to $f$ and $\mathfrak{H}_{2}$ is 2 -dimensional Hausdorff measure. Finally, if $T$ is an open subset of $\Omega$ and $\omega$ a 3 -form, then $\int_{T} \omega$ is equal to the usual integral of the corresponding function with respect to Lebesgue measure.

Continuing with the identification of forms on $\mathbb{R}^{3}$ with functions and vector fields, we find that $d_{0}=\operatorname{grad}, d_{1}=$ curl, $d_{2}=\operatorname{div}, \kappa_{3}$ is multiplication of a scalar field by $x$ to get a vector field, $\kappa_{2}$ takes the cross product of a vector field with $x$ to produce another vector field, and $\kappa_{1}$ takes the dot product of a vector field with $x$. Thus the differential complexes discussed above can be written as follows.

The smooth de Rham complex:

$$
\mathbb{R} \hookrightarrow C^{\infty}(\Omega) \stackrel{\text { grad }}{\longrightarrow} C^{\infty}\left(\Omega ; \mathbb{R}^{3}\right) \stackrel{\text { curl }}{\longrightarrow} C^{\infty}\left(\Omega ; \mathbb{R}^{3}\right) \stackrel{\text { div }}{\longrightarrow} C^{\infty}(\Omega) \rightarrow 0
$$

The $L^{2}$ de Rham complex:

$$
\mathbb{R} \hookrightarrow H^{1}(\Omega) \stackrel{\text { grad }}{\longrightarrow} H\left(\operatorname{curl}, \Omega ; \mathbb{R}^{3}\right) \stackrel{\text { curl }}{\longrightarrow} H\left(\operatorname{div}, \Omega ; \mathbb{R}^{3}\right) \stackrel{\text { div }}{\longrightarrow} L^{2}(\Omega) \rightarrow 0
$$

The polynomial de Rham complex:

$$
\mathbb{R} \hookrightarrow \mathcal{P}_{r}(\Omega) \stackrel{\text { grad }}{\longrightarrow} \mathcal{P}_{r-1}\left(\Omega ; \mathbb{R}^{3}\right) \stackrel{\text { curl }}{\longrightarrow} \mathcal{P}_{r-2}\left(\Omega ; \mathbb{R}^{3}\right) \stackrel{\text { div }}{\longrightarrow} \mathcal{P}_{r-3}(\Omega) \rightarrow 0
$$

The Koszul complex:

$$
0 \rightarrow \mathcal{P}_{r-3}(\Omega) \stackrel{x}{\longrightarrow} \mathcal{P}_{r-2}\left(\Omega ; \mathbb{R}^{3}\right) \stackrel{\times x}{\longrightarrow} \mathcal{P}_{r-1}\left(\Omega ; \mathbb{R}^{3}\right) \stackrel{\cdot x}{\longrightarrow} \mathcal{P}_{r}(\Omega) \rightarrow \mathbb{R} \rightarrow 0
$$

The Hodge Laplacian on 0 -forms and 3 -forms is the ordinary Laplacian $\Delta=$ div grad viewed as an unbounded operator on $L^{2}(\Omega)$ with certain boundary conditions imposed in its domain (basically, Neumann conditions in the case of 0 -forms and Dirichlet conditions in the case of 3-forms). Similarly, the Hodge Laplacian on 1-forms and 2-forms gives the vector Laplacian curl curl - grad div with two different sets of boundary conditions. We will say more on this in Section 6. 
5. Piecewise polynomial differential forms. Let $\mathcal{T}$ be a triangulation by simplices of a domain $\Omega \subset \mathbb{R}^{n}$. In this section we define, in a unified fashion, a variety of finite-dimensional spaces of differential forms on $\Omega$ which are piecewise polynomials with respect to the triangulation $\mathcal{T}$. In the cases where we can identify differential forms with functions and vector fields on $\Omega$, these spaces correspond to well-known finite element spaces, such as the Lagrange space, the Brezzi-Douglas-Marini spaces, the Raviart-Thomas spaces, and the Nedelec spaces of [17, 18].

We begin by describing a set of degrees of freedom for the polynomial spaces $\mathcal{P}_{r} \Lambda^{k}(T)$ and $\mathcal{P}_{r}^{+} \Lambda^{k}(T)$, which will reveal a strong connection between the $\mathcal{P}_{r}$ and $\mathcal{P}_{r}^{+}$spaces. For $T$ a domain in $\mathbb{R}^{n}, 0 \leq k \leq n$, and $r>0$, an element $\omega \in \mathcal{P}_{r} \Lambda^{k}(T)$ is uniquely determined by the following quantities (see [18] for the case $n=3$ ):

$$
\int_{f} \omega \wedge \nu, \quad \nu \in \mathcal{P}_{r-d-1+k}^{+} \Lambda^{d-k}(f), \quad f \in \Delta_{d}(T), \quad k \leq d \leq n .
$$

(For $r<0$, we interpret $\mathcal{P}_{r}^{+} \Lambda^{k}(T)=\mathcal{P}_{r} \Lambda^{k}(T)=0$.) Notice that for $\omega \in \Lambda^{k}(T)$ and $\nu \in \Lambda^{d-k}(T), \omega \wedge \nu \in \Lambda^{d}(T)$ and hence the integral of $\omega \wedge \nu$ on a $d$-dimensional face of $T$ is a well-defined and natural quantity. Notice also that the degrees of freedom for the space $\mathcal{P}_{r} \Lambda^{k}$ involve the moments on faces weighted by elements of $\mathcal{P}_{r}^{+} \Lambda^{k}$. The reverse is true as well. The degrees of freedom for $\omega \in \mathcal{P}_{r}^{+} \Lambda^{k}(T)$ are the moments

$$
\int_{f} \omega \wedge \nu, \quad \nu \in \mathcal{P}_{r-d+k} \Lambda^{d-k}(f), \quad f \in \Delta_{d}(T), \quad k \leq d \leq n .
$$

This set of degrees of freedom was presented in [15]. See also [17].

In this way, we obtain two families of piecewise polynomial $k$-forms, each indexed by polynomial degree $r$ :

$$
\begin{aligned}
\mathcal{P}_{r} \Lambda^{k}(\mathcal{T}) & =\left\{\omega \in H \Lambda^{k}(\Omega) \mid \omega_{\mid T} \in \mathcal{P}_{r} \Lambda^{k}(T) \quad \forall T \in \mathcal{T}\right\}, \\
\mathcal{P}_{r}^{+} \Lambda^{k}(\mathcal{T}) & =\left\{\omega \in H \Lambda^{k}(\Omega) \mid \omega_{\left.\right|_{T}} \in \mathcal{P}_{r}^{+} \Lambda^{k}(T) \quad \forall T \in \mathcal{T}\right\} .
\end{aligned}
$$

We believe these should be regarded as the most natural finite element approximations of the Sobolev differential form spaces $H \Lambda^{k}(\Omega)$. This is certainly true in $n=1$ dimension, where for each $r \geq 0$ and partition of the domain, we obtain a unique finite element discretization of $H^{1}(\Omega)$ and of $L^{2}(\Omega)$ : the $\mathcal{P}_{r+1}^{\text {cont }}$ space of continuous piecewise polynomials of degree $r+1$ and the $\mathcal{P}_{r}$ space of all piecewise polynomials of degree $r$, respectively. For $k=0$ in any number of dimensions, then

$$
\mathcal{P}_{r}^{+} \Lambda^{0}(\mathcal{T})=\mathcal{P}_{r+1} \Lambda^{0}(\mathcal{T})
$$

is the usual Lagrange space of all continuous piecewise polynomials of degree $r+1$, the most natural discretization of $H^{1}(\Omega) \cong H \Lambda^{0}(\Omega)$. For $k=n$, we get

$$
\mathcal{P}_{r}^{+} \Lambda^{n}(\mathcal{T})=\mathcal{P}_{r} \Lambda^{n}(\mathcal{T})
$$


is the space of all piecewise polynomials of degree $r$, the most natural discretization of $L^{2}(\Omega) \cong H \Lambda^{n}(\Omega)$. For $k=n-1$, we may identify $H \Lambda^{n-1}(\Omega)$ with $H\left(\operatorname{div}, \Omega ; \mathbb{R}^{n}\right)$, and $\mathcal{P}_{r}^{+} \Lambda^{n-1}(\mathcal{T})$ is the Raviart-Thomas space of index $r$ and $\mathcal{P}_{r} \Lambda^{n-1}(\mathcal{T})$ the Brezzi-Douglas-Marini space of index $r$, the best known discretizations of $H\left(\right.$ div). Finally, for $k=1, n=3, H \Lambda^{1}(\Omega)$ can be identified with $H\left(\operatorname{curl}, \Omega ; \mathbb{R}^{3}\right)$ and $\mathcal{P}_{r}^{+} \Lambda^{1}(\mathcal{T})$ and $\mathcal{P}_{r} \Lambda^{1}(\mathcal{T})$ are the Nedelec finite element spaces of the first and second kind, respectively, the best known spaces of $H$ (curl) elements, illustrated in Figure 6 .
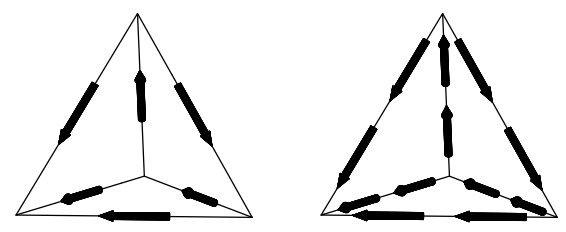

FIG. 6. Lowest order Nedelec H(curl) elements of the first kind and the second kind.

These spaces fit together to provide a number of piecewise polynomial analogues of the de Rham complex. For any $r \geq 0$, we have the complex

$$
\mathbb{R} \hookrightarrow \mathcal{P}_{r}^{+} \Lambda^{0}(\mathcal{T}) \stackrel{d}{\longrightarrow} \mathcal{P}_{r}^{+} \Lambda^{1}(\mathcal{T}) \stackrel{d}{\longrightarrow} \cdots \stackrel{d}{\longrightarrow} \mathcal{P}_{r}^{+} \Lambda^{n}(\mathcal{T}) \rightarrow 0 .
$$

In case $r=0$, this is the complex of piecewise polynomial forms introduced by Whitney to calculate de Rham cohomology [20]. It has the same cohomology spaces as the smooth de Rham complex, so, in particular, is exact if $\Omega$ is contractible. The connection betweens Whitney's forms and mixed finite elements was recognized by Bossavit [6]. Using an element diagram to stand in for the corresponding finite element space, in $n=3$ dimensions the complex of Whitney forms may be represented

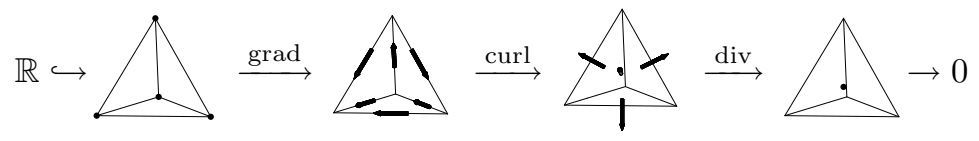

The degrees of freedom in (5.1) and (5.2) determine projection operators $\Pi_{r}^{k}: \Lambda^{k}(\Omega) \rightarrow \mathcal{P}_{r} \Lambda^{k}(\mathcal{T})$ and $\Pi_{r+}^{k}: \Lambda^{k}(\Omega) \rightarrow \mathcal{P}_{r}^{+} \Lambda^{k}(\mathcal{T})$ respectively. These may be used to relate the smooth de Rham complex (4.2) to the piecewise polynomial de Rham complex (5.3). Namely, the following diagram commutes:

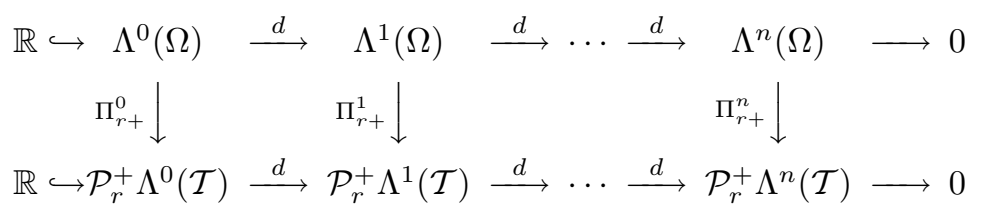


Another piecewise polynomial differential complex with the same cohomology uses the $\mathcal{P}_{r} \Lambda^{k}$ spaces:

$$
\mathbb{R} \hookrightarrow \mathcal{P}_{r+n} \Lambda^{0}(\mathcal{T}) \stackrel{d}{\longrightarrow} \mathcal{P}_{r+n-1} \Lambda^{1}(\mathcal{T}) \stackrel{d}{\longrightarrow} \cdots \stackrel{d}{\longrightarrow} \mathcal{P}_{r} \Lambda^{n}(\mathcal{T}) \rightarrow 0
$$

The complex (5.3) is a subcomplex of (5.4), in the sense that each space occurring in the former complex is a subspace of the corresponding space in the latter complex. The complex (5.4) appears, generalized to the case of degree varying by the element, in [13]. Note that this complex ends with the same space $\mathcal{P}_{r} \Lambda^{n}(\Omega)=\mathcal{P}_{r}^{+} \Lambda^{n}(\Omega)$ as (5.4), but in contrast with (5.3) the degree index $r$ decreases with increasing differential form order $k$.

In one dimension the two complexes (5.3), (5.4) coincide, but in two dimensions they are distinct. In $n>2$ dimensions there are additional piecewise polynomial complexes which can be built from the same $\mathcal{P}_{r} \Lambda^{k}$ and $\mathcal{P}_{r} \Lambda^{k+1}$ spaces, have the same cohomology, and end in the same space $\mathcal{P}_{r} \Lambda^{0}(\mathcal{T})$. These are intermediate between (5.3) and (5.4), and strictly ordered by the subcomplex relationship. Specifically, there are $2^{n-1}$ such piecewise polynomials complexes in $n$ dimensions. In three dimensions the other two are

$$
\mathbb{R} \hookrightarrow \mathcal{P}_{r+2} \Lambda^{0}(\mathcal{T}) \stackrel{d}{\longrightarrow} \mathcal{P}_{r+1} \Lambda^{1}(\mathcal{T}) \stackrel{d}{\longrightarrow} \mathcal{P}_{r}^{+} \Lambda^{2}(\mathcal{T}) \stackrel{d}{\longrightarrow} \mathcal{P}_{r} \Lambda^{3}(\mathcal{T}) \rightarrow 0
$$

and

$$
\mathbb{R} \hookrightarrow \mathcal{P}_{r+2} \Lambda^{0}(\mathcal{T}) \stackrel{d}{\longrightarrow} \mathcal{P}_{r+1}^{+} \Lambda^{1}(\mathcal{T}) \stackrel{d}{\longrightarrow} \mathcal{P}_{r+1} \Lambda^{2}(\mathcal{T}) \stackrel{d}{\longrightarrow} \mathcal{P}_{r} \Lambda^{3}(\mathcal{T}) \rightarrow 0
$$

6. Differential complexes and stability. Let $\Omega$ be a contractible subdomain of $\mathbb{R}^{n}$ and $0 \leq k \leq n$ an integer. Given $f \in L^{2} \Lambda^{k}(\Omega)$, define $\mathcal{L}: H \Lambda^{k-1}(\Omega) \times H \Lambda^{k}(\Omega) \rightarrow \mathbb{R}$ by

$$
\mathcal{L}(\tau, v)=\int_{\Omega}\left(\frac{1}{2}\langle\tau, \tau\rangle-\langle d \tau, v\rangle-\frac{1}{2}\langle d v, d v\rangle+\langle f, v\rangle\right) d x
$$

where the angular brackets indicate the inner product of forms as defined in (4.1). Then $\mathcal{L}$ admits a unique critical point, a saddle point, $(\sigma, u) \in$ $H \Lambda^{k-1}(\Omega) \times H \Lambda^{k}(\Omega)$ determined by the equations

$$
\begin{gathered}
\int_{\Omega}\langle\sigma, \tau\rangle d x=\int_{\Omega}\langle d \tau, u\rangle d x \quad \forall \tau \in H \Lambda^{k-1}(\Omega), \\
\int_{\Omega}\langle d \sigma, v\rangle d x+\int_{\Omega}\langle d u, d v\rangle d x=\int_{\Omega}\langle f, v\rangle d x \quad \forall v \in H \Lambda^{k}(\Omega) .
\end{gathered}
$$

These are weak formulations of the equations

$$
\sigma=d^{*} u, \quad d \sigma+d^{*} d u=f,
$$

respectively, and hence together, give the Hodge Laplacian problem $\left(d d^{*}+\right.$ $\left.d^{*} d\right) u=f$. Implied as well are the natural boundary conditions that the 
trace of $u$ as a $k$-form on $\partial \Omega$ and the trace of $d u$ as a $(k+1)$-form on $\partial \Omega$ both must vanish.

Let us consider more concretely the situation in $n=3$ dimensions, identifying the spaces $L^{2} \Lambda^{k}(\Omega)$ with function spaces as described at the end of Section 4. For $k=3,(6.1),(6.2)$ become: find $\sigma \in H\left(\operatorname{div}, \Omega ; \mathbb{R}^{3}\right)$, $u \in L^{2}(\Omega)$ such that

$$
\begin{gathered}
\int_{\Omega} \sigma \cdot \tau d x=\int_{\Omega} \operatorname{div} \tau u d x \quad \forall \tau \in H\left(\operatorname{div}, \Omega ; \mathbb{R}^{3}\right), \\
\int_{\Omega} \operatorname{div} \sigma v d x=\int_{\Omega} f v d x \quad \forall v \in L^{2}(\Omega),
\end{gathered}
$$

i.e., the weak formulation of the steady state heat conduction problem (with unit resistivity) discussed in Section 1. This is the standard mixed formulation for the Dirichlet problem for the Poisson equation: (6.3) is equivalent to the differential equation $\sigma=-\operatorname{grad} u$ and the boundary condition $u=0$, while (6.4) is equivalent to $\operatorname{div} \sigma=f$.

For $k=2$, the unknowns $\sigma \in H\left(\operatorname{curl}, \Omega ; \mathbb{R}^{3}\right)$ and $u \in H\left(\operatorname{div}, \Omega ; \mathbb{R}^{3}\right)$ satisfy the differential equations

$$
\sigma=\operatorname{curl} u, \quad \operatorname{curl} \sigma-\operatorname{grad} \operatorname{div} u=f,
$$

and the boundary conditions $u \times n=0, \operatorname{div} u=0$ on $\partial \Omega$, so this is a mixed formulation for the vectorial Poisson equation

$$
\text { (curl curl - grad div) } u=f
$$

with the auxiliary variable $\sigma=\operatorname{curl} u$. For $k=1,(6.1),(6.2)$ is a different mixed formulation of the vectorial Poisson equation (6.5). Now $\sigma \in H^{1}(\Omega)$ and $u \in H\left(\operatorname{curl}, \Omega ; \mathbb{R}^{3}\right)$ satisfy

$$
\sigma=-\operatorname{div} u, \quad(\operatorname{grad} \sigma+\operatorname{curl} \operatorname{curl}) u=f,
$$

with boundary conditions $u \cdot n=0,(\operatorname{curl} u) \times n=0$.

Finally, we interpret the case $k=0$. Here, in view of the $L^{2}$ de Rham sequence, we interpret $H \Lambda^{-1}(\Omega)$ as $\mathbb{R}$ with the operator $H \Lambda^{-1}(\Omega) \rightarrow$ $H \Lambda^{0}(\Omega)$ just the inclusion of $\mathbb{R}$ in $H^{1}(\Omega)$. Thus, the unknowns are $\sigma \in \mathbb{R}$ and $u \in H^{1}(\Omega)$, (6.1) just gives the equation $\sigma=\int_{\Omega} u d x /$ meas $(\Omega)$, while (6.2) is

$$
\int_{\Omega} \operatorname{grad} u \cdot \operatorname{grad} v d x+\sigma \int_{\Omega} v d x=\int_{\Omega} f v d x \quad \forall v \in H^{1}(\Omega) .
$$

Thus we just have the usual weak formulation of the Neumann problem for the Poisson equation (if $\int_{\Omega} f d x=0$, then $\sigma=0$ ).

Returning now to the case of general $n$, suppose we are given a triangulation, and let

$$
\mathbb{R} \hookrightarrow \Lambda_{h}^{0} \stackrel{d_{h}}{\longrightarrow} \Lambda_{h}^{1} \stackrel{d_{h}}{\longrightarrow} \cdots \stackrel{d_{h}}{\longrightarrow} \Lambda_{h}^{n} \rightarrow 0
$$


denote any of the $2^{n-1}$ piecewise polynomial de Rham complexes discussed in Section 5, e.g., (5.3) or (5.4). Here we use $d_{h}$ to denote the restriction of the exterior differential $d$, and we shall denote by $d_{h}^{*}$ its adjoint. We further suppose we have a commuting diagram of the form

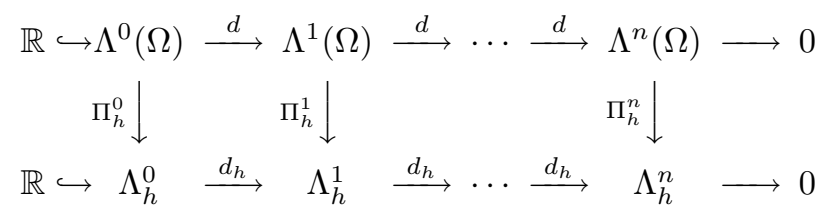

We shall demonstrate stability of the finite element method: find $\sigma \in$ $\Lambda_{h}^{k-1}, u \in \Lambda_{h}^{k}$ such that

$$
\begin{gathered}
\int_{\Omega}\langle\sigma, \tau\rangle d x=\int_{\Omega}\langle d \tau, u\rangle d x \quad \forall \tau \in \Lambda_{h}^{k-1} \\
\int_{\Omega}\langle d \sigma, v\rangle d x+\int_{\Omega}\langle d u, d v\rangle d x=\int_{\Omega}\langle f, v\rangle d x \quad \forall v \in \Lambda_{h}^{k} .
\end{gathered}
$$

Let $B:\left[H \Lambda^{k-1}(\Omega) \times H \Lambda^{k}(\Omega)\right] \times\left[H \Lambda^{k-1}(\Omega) \times H \Lambda^{k}(\Omega)\right] \rightarrow \mathbb{R}$ denote the bounded bilinear form

$$
B(\sigma, u ; \tau, v)=\int_{\Omega}(\langle\sigma, \tau\rangle-\langle d \tau, u\rangle+\langle d \sigma, v\rangle+\langle d u, d v\rangle) d x .
$$

Stability of the method (6.8), (6.9) is equivalent to the inf-sup condition for $B$ restricted to the finite element spaces [4]. That is, we must establish the existence of constants $\gamma>0, C<\infty$ such that for any $(\sigma, u) \in \Lambda_{h}^{k-1} \times \Lambda_{h}^{k}$ there exists $(\tau, v) \in \Lambda_{h}^{k-1} \times \Lambda_{h}^{k}$ with

$$
\begin{gathered}
B(\sigma, u ; \tau, v) \geq \gamma\left(\|\sigma\|_{H \Lambda^{k-1}}^{2}+\|u\|_{H \Lambda^{k}}^{2}\right), \\
\|\tau\|_{H \Lambda^{k-1}}+\|v\|_{H \Lambda^{k}} \leq C\left(\|\sigma\|_{H \Lambda^{k-1}}+\|u\|_{H \Lambda^{k}}\right) .
\end{gathered}
$$

To do so, we first establish some preliminary results.

Lemma 6.1. Given $u \in \Lambda_{h}^{k}$, there exist unique forms $\rho \in d_{h}^{*}\left(\Lambda_{h}^{k}\right) \subset$ $\Lambda_{h}^{k-1}$ and $\phi \in d_{h}\left(\Lambda_{h}^{k}\right) \subset \Lambda_{h}^{k+1}$ with

$$
\begin{gathered}
u=d_{h} \rho+d_{h}^{*} \phi, \quad d_{h}^{*} \rho=0, \quad d_{h} \phi=0, \\
\|u\|^{2}=\left\|d_{h} \rho\right\|^{2}+\left\|d_{h}^{*} \phi\right\|^{2} .
\end{gathered}
$$

Proof. This is a special case of a more general result. Let

$$
0 \rightarrow X \stackrel{f}{\longrightarrow} Y \stackrel{g}{\longrightarrow} Z \rightarrow 0
$$

be a short exact sequence where $X, Y$, and $Z$ are finite-dimensional Hilbert spaces and $f$ and $g$ linear maps. Then $Y$ decomposes into orthogonal 
summands $A:=\mathcal{R}(f)=\mathcal{N}(g)$ and $B:=\mathcal{N}\left(f^{*}\right)=\mathcal{R}\left(g^{*}\right)$. Thus any $y \in Y$ may be decomposed as $y=f x+g^{*} z$ for some unique $x \in X, z \in Z$, and we have $\|y\|_{Y}^{2}=\|f x\|_{Y}^{2}+\left\|g^{*} z\right\|_{Y}^{2}$. We apply these results with $Y=\Lambda_{h}^{k}$, $Z=d_{h}\left(\Lambda_{h}^{k}\right) \subset \Lambda_{h}^{k+1}$, and $X=d_{h}^{*}\left(\Lambda_{h}^{k}\right) \subset \Lambda_{h}^{k-1}$.

LEMmA 6.2. Suppose that for any $u \in \Lambda_{h}^{k}$ of the form (6.12),

$$
\left\|d_{h}^{*} \phi\right\| \leq K\left\|d_{h} u\right\|, \quad\|\rho\| \leq K^{\prime}\left\|d_{h} \rho\right\|,
$$

where $K$ and $K^{\prime}$ are constants independent of $\rho, \phi$, and $h$. Then the stability conditions (6.10) and (6.11) are satisfied.

Proof. Let $\tau=\sigma-t \rho \in \Lambda_{h}^{k-1}$ and $v=u+d_{h} \sigma \in \Lambda_{h}^{k}$ with $t=1 /\left(K^{\prime}\right)^{2}$. Using (6.13), the hypotheses of the lemma, and a simple computation, we get

$$
\begin{aligned}
B(\sigma, u ; \tau, v) & =\|\sigma\|^{2}+\left\|d_{h} \sigma\right\|^{2}+\left\|d_{h} u\right\|^{2}+t\left\|d_{h} \rho\right\|^{2}-t \int_{\Omega}\langle\sigma, \rho\rangle d x \\
& \geq \frac{1}{2}\|\sigma\|^{2}+\left\|d_{h} \sigma\right\|^{2}+\left\|d_{h} u\right\|^{2}+t\left\|d_{h} \rho\right\|^{2}-\frac{t^{2}}{2}\|\rho\|^{2} \\
& \geq \frac{1}{2}\|\sigma\|^{2}+\left\|d_{h} \sigma\right\|^{2}+\left\|d_{h} u\right\|^{2}+\left\|d_{h} \rho\right\|^{2}\left(t-t^{2}\left(K^{\prime}\right)^{2} / 2\right) \\
& \geq \frac{1}{2}\|\sigma\|^{2}+\left\|d_{h} \sigma\right\|^{2}+\frac{1}{2}\left\|d_{h} u\right\|^{2}+\frac{1}{2\left(K^{\prime}\right)^{2}}\left\|d_{h} \rho\right\|^{2}+\frac{1}{2 K^{2}}\left\|d_{h}^{*} \phi\right\|^{2} \\
& \geq \frac{1}{2}\|\sigma\|^{2}+\left\|d_{h} \sigma\right\|^{2}+\frac{1}{2}\left\|d_{h} u\right\|^{2}+\frac{1}{2\left(K^{\prime \prime}\right)^{2}}\|u\|^{2},
\end{aligned}
$$

where $K^{\prime \prime}=\max \left(K^{\prime}, K\right)$. Hence, we obtain (6.10) with $\gamma>0$ depending only on $K$ and $K^{\prime}$. The upper bound (6.11) follows from the fact that

$$
\|\rho\| \leq K^{\prime}\left\|d_{h} \rho\right\| \leq K^{\prime}\|u\| .
$$

The hypotheses of the lemma are easily seen to be valid if we allow the constants $K$ and $K^{\prime}$ to depend on $h$, with $K$ the norm of the inverse of $d_{h}$ restricted to the orthogonal complement of its kernel in $\Lambda_{h}^{k}$ and $K^{\prime}$ is the norm of the inverse of $d_{h}$ restricted to the orthogonal complement of its kernel in $\Lambda_{h}^{k-1}$. To show that the constants $K$ and $K^{\prime}$ can be taken independent of $h$, we need to make use of approximation properties of the interpolation operators $\Pi_{h}^{k}$ and elliptic regularity of appropriately chosen boundary value problems. We shall assume that for $u \in H^{1} \Lambda^{k-1}(\Omega)$ with $d u \in \Lambda_{h}^{k}$,

$$
\left\|u-\Pi_{h}^{k-1} u\right\| \leq C h\|u\|_{1} .
$$

We note that the condition $d u \in \Lambda_{h}^{k}$ is needed in some cases for the interpolant $\Pi_{h}^{k-1} u$ to be defined. We next consider boundary value problems 
of the form: Given $\sigma_{h} \in \Lambda_{h}^{k-1}$, find $(\sigma, u) \in H \Lambda^{k-1}(\Omega) \times d H \Lambda^{k-1}(\Omega)$ determined by the equations

$$
\begin{array}{cc}
\int_{\Omega}\langle\sigma, \tau\rangle d x & =\int_{\Omega}\langle d \tau, u\rangle d x \quad \forall \tau \in H \Lambda^{k-1}(\Omega), \\
\int_{\Omega}\langle d \sigma, v\rangle d x=\int_{\Omega}\left\langle d_{h} \sigma_{h}, v\right\rangle d x & \forall v \in d H \Lambda^{k-1}(\Omega) .
\end{array}
$$

This is a weak formulation of the equations

$$
\sigma=d^{*} u, \quad d \sigma=d_{h} \sigma_{h}, \quad d u=0,
$$

together with the natural boundary condition that the trace of $u$ as a $k$-form on $\partial \Omega$ vanishes. We shall assume that the solution satisfies the regularity estimate:

$$
\|\sigma\|_{1} \leq C\left\|d_{h} \sigma_{h}\right\| .
$$

We apply this result first in the case when $\sigma_{h}=\rho$. Since $d_{h}^{*} \rho=0$, there exists $u_{h} \in d_{h} \Lambda_{h}^{k-1}$ such that $\rho=d_{h}^{*} u_{h}$, i.e.,

$$
\int_{\Omega}\langle\rho, \tau\rangle d x=\int_{\Omega}\left\langle d \tau, u_{h}\right\rangle d x \quad \forall \tau \in \Lambda_{h}^{k-1} .
$$

Since $d \sigma=d_{h} \sigma_{h} \in \Lambda_{h}^{k}$, we have by the commuting diagram (6.7) that $d \Pi_{h}^{k-1} \sigma=\Pi_{h}^{k} d \sigma=d \sigma=d_{h} \sigma_{h}=d_{h} \rho$. Choosing $\tau=\rho-\Pi_{h}^{k-1} \sigma$, we get

$$
\|\rho\| \leq\left\|\Pi_{h}^{k-1} \sigma\right\| \leq\|\sigma\|+\left\|\sigma-\Pi_{h}^{k-1} \sigma\right\| \leq C\|\sigma\|_{1} \leq\left\|d_{h} \rho\right\| .
$$

To establish the first inequality of the lemma, it is enough to show that $\|\phi\| \leq C\left\|d_{h}^{*} \phi\right\|$, since

$$
\left\|d_{h}^{*} \phi\right\|^{2}=\left(\phi, d_{h} d_{h}^{*} \phi\right)=\left(\phi, d_{h} u\right) \leq\|\phi\|\left\|d_{h} u\right\| .
$$

Because $d_{h} \phi=0$, we can write $\phi=d_{h} w, w \in \Lambda_{h}^{k}$. We then apply our regularity result in the case when $\sigma_{h}=w$ and $k$ is replaced by $k+1$. Hence, $\|\sigma\|_{1} \leq C\left\|d_{h} w\right\| \leq C\|\phi\|$. Since $d \sigma=d_{h} \sigma_{h} \in \Lambda_{h}^{k+1}$, we again use the commuting diagram (6.7) to write

$$
d \Pi_{h}^{k} \sigma=\Pi_{h}^{k+1} d \sigma=d \sigma=d_{h} \sigma_{h}=d_{h} w=\phi .
$$

Now

$$
\|\phi\|^{2}=\left(d \Pi_{h}^{k} \sigma, \phi\right)=\left(\Pi_{h}^{k} \sigma, d_{h}^{*} \phi\right) \leq\left\|\Pi_{h}^{k} \sigma\right\|\left\|d_{h}^{*} \phi\right\| .
$$

But

$$
\left\|\Pi_{h}^{k} \sigma\right\| \leq\|\sigma\|+\left\|\sigma-\Pi_{h}^{k} \sigma\right\| \leq C\|\sigma\|_{1} \leq C\|\phi\| .
$$

Combining these results establishes the first inequality. 


\section{REFERENCES}

[1] D. N. ARnold, Differential complexes and numerical stability, in Proceedings of the International Congress of Mathematicians, Vol. I: Plenary Lectures and Ceremonies, L. Tatsien, ed., Higher Education Press, Beijing, 2002, pp. 137157.

[2] D. N. Arnold And R. Winther, Mixed finite elements for elasticity, Numer. Math., 92 (2002), pp. 401-419.

[3] V. I. Arnold, Mathematical Methods of Classical Physics, Springer-Verlag, New York, 1978.

[4] I. BABUŠKA AND A. K. AzIZ, Survey lectures on the mathematical foundations of the finite element method, in The Mathematical Foundations of the Finite Element Method with Applications to Partial Differential Equations, Academic Press, New York, 1972.

[5] I. BabušKa And R. Narasimhan, The Babuška-Brezzi condition and the patch test: an example, Comput. Methods Appl. Mech. Engrg., 140 (1997), pp. 183199.

[6] A. Bossavit, Whitney forms: a class of finite elements for three-dimensional computations in electromagnetism, IEEE Proc. A, 135 (1988), pp. 493-500.

[7] F. BREzZI, On the existence, uniqueness and approximation of saddle-point problems arising from Lagrangian multipliers, Rev. Française Automat. Informat. Recherche Opérationnelle Sér. Rouge, 8 (1974), pp. 129-151.

[8] F. Brezzi and K.-J. Bathe, A discourse on the stability conditions for mixed finite element formulations, Comput. Methods Appl. Mech. Engrg., 82 (1990), pp. 27-57. Reliability in computational mechanics (Austin, TX, 1989).

[9] F. Brezzi, J. Douglas, Jr., R. Durán, and M. Fortin, Mixed finite elements for second order elliptic problems in three variables, Numer. Math., 51 (1987).

[10] F. Brezzi, J. Douglas, JR., And L. D. Marini, Two families of mixed finite elements for second order elliptic problems, Numer. Math., 47 (1985), pp. $217-$ 235.

[11] F. Brezzi And M. Fortin, Mixed and hybrid finite element methods, SpringerVerlag, New York, 1991.

[12] P. G. Ciarlet, The finite element method for elliptic problems, North-Holland, Amsterdam, 1978.

[13] L. Demkowicz, P. Monk, W. Rachowicz, and L. Vardapetyan, De Rham diagram for hp finite element spaces, Computers \& Mathematics with Applications, 39 (2000), pp. 29-38.

[14] V. Girault And P.-A. Raviart, Finite element methods for Navier-Stokes equations, Springer-Verlag, New York, 1986.

[15] R. Hiptmair, Canonical construction of finite elements, Math. Comp., 68 (1999), pp. $1325-1346$.

[16] — Finite elements in computational electromagnetism, Acta Numerica, 11 (2002), pp. 237-340.

[17] J.-C. NÉDÉLEC, Mixed finite elements in $\mathbf{R}^{3}$, Numer. Math., 35 (1980), pp. 315 341.

[18] — A new family of mixed finite elements in $\mathbf{R}^{3}$, Numer. Math., 50 (1986), pp. $57-81$.

[19] P.-A. Raviart and J. M. Thomas, A mixed finite element method for 2nd order elliptic problems, in Mathematical aspects of finite element methods (Proc. Conf., Consiglio Naz. delle Ricerche (C.N.R.), Rome, 1975), Springer, Berlin, 1977, pp. 292-315. Lecture Notes in Math., Vol. 606.

[20] H. Whitney, Geometric Integration Theory, Princeton University Press, Princeton, 1957. 